\title{
Optimal Genetic Augmentation Strategies for a Threatened Species using a Continent-Island Model
}

\author{
Erin N. Bodine ${ }^{1, *}$, Marco V. Martinez ${ }^{2}$
}

\begin{abstract}
One conservation method of reducing species loss is to augment a declining/threatened wild population with individuals from a captive-bred or stable, wild population. This method is known as species augmentation. We have modeled the change in the frequency of a detrimental allele in a threatened population using the continent-island genetic population model. We use optimal control theory to determine augmentation strategies which minimize the presence of the detrimental allele in an endangered population in minimum time while minimizing the cost of augmenting the endangered population. We present the construction of the optimal control formulation, the necessary conditions for an optimal control, the characterization of an optimal control, the algorithm for computing numerical solutions, and some numerical simulations. Additionally, we discuss some of the challenges of systematically exploring the effects of uncertain parameters in time minimizing optimal control problems and demonstrate one method for quantifying the sensitivity of the optimal control strategy with respect to uncertain parameter values.
\end{abstract}

Keywords: optimal control theory, sensitivity analysis, species augmentation, population genetics, continent-island model

\section{Introduction}

Over the past four decades a significant proportion of ecology research has focused on studying and preserving biodiversity. More recently, there has also been interest in studying and preserving the genetic diversity within single populations. One conservation method of reducing species loss is to augment a declining/threatened wild population with individuals from a captive-bred or stable, wild population. This method is known as species augmentation. The term genetic augmentation is applied when the augmentation is specifically designed to alter the genetic diversity of the threatened population. A variety of population studies of specific threatened species have recommended the use of augmentation (often in conjunction with other conservation policies) as a means of preventing extinction and/or promoting population growth. See $[10,12,13,15,18,24]$ for some examples.

An example of an augmentation project that took genetic factors into consideration is the Florida panther augmentation. In the 1980s and early 1990s the Florida panther (Puma concolor coryi) population had been reduced to roughly 20-30 individuals (in the wild) $[2,23]$ due to a long history of legal hunting of Florida panthers and several decades of habitat loss [1, 23]. In 1995, eight female Texas panthers (Puma concolor stanleyana) of

\footnotetext{
${ }^{1}$ Department of Mathematics \& Computer Science, Rhodes College, Memphis, TN

${ }^{2}$ Department of Mathematics, North Central College, Naperville, IL

* Correspondence: bodinee@rhodes.edu
} 
breeding age were introduced into the Florida panther population in an effort to increase low genetic diversity which was causing genetic defects such as kinked tails, heart defects, and one or both testicles not descending in mature males [16, 23]. In 1995 Hedrick used the Continent-Island model and an adaptation of the Continent-Island model which included the effects of selection to examine the genetic restoration of the endangered Florida panther by augmenting the dwindling population with female Texas panthers [13].

The standard Continent-Island model in population genetics was originally proposed by Seawell Wright in the 1930s [26]; it tracks the change in the allele frequency of an isolated (island) population due to gene flow from a large source (continent) population [14, 26]. Although gene flow may generally occur in both directions between two populations, in the Continent-Island model it is assumed that any migration from the island to the continent has a negligible effect on the continent allele frequencies. Additionally, the Continent-Island model assumes the allele frequencies of the isolated population are not changing due to other causes like natural selection, genetic drift, non-random mate selection, etc.

Suppose we are interested in a gene with two alleles $A_{1}$ and $A_{2}$. Let $q_{t}$ be the frequency of $A_{2}$ (which we assume to be the recessive allele) in the isolated population in generation $t$ after the source population migrates into the isolated population. Let $\hat{q}$ be the frequency of $A_{2}$ in the source population, and we assume this frequency is constant. Then the ContinentIsland model is given by

$$
q_{t+1}=\left(\begin{array}{ll}
1 & \alpha
\end{array}\right) q_{t}+\alpha \hat{q}=q_{t} \quad \alpha\left(q_{t} \quad \hat{q}\right)
$$

where $\alpha$ is the proportion of the island population which are migrants from the continent (thus $0 \quad \alpha<1$ ). Note, we assume the genotypes of the migrants in each generation are in proportion with the genotypes of the entire continent population. Offspring born from migrant/migrant or migrant/resident matings are both considered island residents.

It can be shown, using induction that

$$
q_{t}=\left(\begin{array}{llll}
1 & \alpha
\end{array}\right)^{t} q_{0}+1 \quad\left(\begin{array}{ll}
1 & \alpha
\end{array}\right)^{t} \hat{q} .
$$

Notice, if $\alpha=0$, then $q_{t}=q_{0}$ for all $t$, and if $0<\alpha<1$ then $q_{t} \quad \hat{q}$ as $t \quad$.

Hedrick used the Continent-Island model to evaluate specific augmentation policy recommendations for the Florida panther and determine the conditions under which the genetic restoration would be successful [13]. Since then Bodine et al. have used optimal control theory to determine optimal species augmentation strategies for threatened and endangered populations using continuous and discrete models $[5,6]$. However, the models used by Bodine et al. did not simulate population genetics. In each of the models presented by Bodine et al. it was assumed that at each time step natural resource managers could control the rate of movement from the source population to the endangered population.

The remainder of the paper explores the use of optimal control theory in generating optimal genetic augmentation strategies for a threatened population. In Section 2, an optimal control formulation using the Continent-Island model is constructed, the existence of an optimal control solution is proven, the necessary conditions for an optimal control solution are derived, and the characterization of an optimal control is found. Section 3, describes the numerical methods used to generate an optimal control and corresponding state given a single set of parameter values, and proposes a novel method for exploring how the qualitative optimal control strategy varies with uncertain parameter values. Then, in Section 4 numerical results for a few illustrative parameter sets are shown, and the sensitivity of the qualitative optimal augmentation strategy to the parameters values is shown and discussed. Lastly, in Section 5 we discuss how this optimal control approach to genetic augmentation is be used to inform augmentation policy and adapted to include more complicated population dynamics. 


\section{Optimal Control Formulation: Controlling One-way Gene Flow}

Consider an isolated and endangered population suffering from the repercussions of a high frequency of a recessive allele with detrimental effects. In an effort to reduce the negative effects of the recessive allele and to preserve the endangered population, individuals from a source population are introduced into the endangered population. Like Hedrick, we can model the frequency of the recessive allele in the endangered population using the Continent-Island model where the island population represents the endangered population and the continent population represents the source population for augmentation. However, we will assume that the number of source population individuals being introduced into the endangered population at each time step can be controlled, as it would be a species augmentation project. The objective in controlling the number of individuals augmenting the endangered population at each time step is to minimize the time it takes to lower the frequency of the recessive allele within the endangered population to the frequency of the recessive allele in the source population while also minimizing the number of individuals which have to be translocated into the endangered population, thus minimizing the cost of the augmentation project. We use optimal control theory to analyze this problem.

Define the set

$$
\Omega=(\alpha, T) \quad \alpha=\left[\alpha_{0}, \alpha_{1}, \alpha_{2}, \ldots, \alpha_{T}{ }_{1}\right], 0 \quad \alpha_{t} \quad \alpha_{\max }<1 \text { for all } t, \text { and } T \quad \mathbb{Z}^{+} .
$$

We wish to minimize the objective functional

$$
J(\alpha, T)=C_{0}\left(q_{T} \quad \hat{q}\right)^{2}+{ }_{t=0}^{T 1} C_{1} \alpha_{t}^{2}+C_{2} \alpha_{t}+C_{3}(T+1)
$$

over the set $\Omega$ subject to the state equation

$$
q_{t+1}=\left(\begin{array}{ll}
1 & \alpha_{t}
\end{array}\right) q_{t}+\alpha_{t} \hat{q}
$$

where $q_{t}$ is the frequency of the recessive allele in the endangered population in generation $t$, $q_{0}$ is the (known) initial frequency of the recessive allele in the endangered population, $\hat{q}$ is the (constant) frequency of the recessive allele in the source population, $\alpha_{t}$ is the proportion of the island population at generation $t$ which are translocated from a source population, $C_{1}$ and $C_{2}$ are non-negative cost coefficients (with at least one coefficient being non-zero), and $C_{0}$ and $C_{3}$ are positive coefficients which balance the relative importance of minimizing the difference between the recessive allele frequency in the endangered and source populations, minimizing the final time, and minimizing the total cost of translocation. Since the endangered population has a high frequency of the recessive allele, we assume $q_{0}$ is close to 1 and that $q_{0}>\hat{q}$. Note, in minimizing the objective functional, we are minimizing the difference between recessive allele frequencies of the endangered and source populations, the total time, and the cost of translocating individuals from the source population into the endangered population.

We assume costs can be both linearly and nonlinearly dependent on the control. Since each $\alpha_{t}$ corresponds to a proportion of the threatened population at time $t$, linear costs correspond to costs of augmentation that increase linearly with the proportion of the threatened population which comes from the source population at each time step. Note costs that would increase linearly with the number of individuals translocated (at each time step), would also increase linearly with respect to the proportion of the threatened population which are translocated individuals (at each time step). An example of such a cost is the average cost for transporting each individual which may include a medical examination and/or various vaccinations. An example of a cost which does not increase linearly with respect to the number of individuals translocated or proportion of the threatened population which are translocated individuals (at each time step) is the cost of finding suitable individuals for 
translocating. Natural resource manager might choose to only translocate individuals of breeding age (as was done in the Florida panther augmentation [16] and the North Cascade grizzly bear augmentation [24]). Since the search time for finding increasing quantities of suitable individuals for augmentation increases at a faster than linear rate, the corresponding cost also increases at a faster than linear rate. Here for simplicity, we represent the nonlinear cost as a quadratic cost.

\subsection{Existence of an Optimal Control}

Theorem 2.1. Given the optimal control formulation in Equations (2)-(3), there exists a pair $(\alpha, T)$ such that $J(\alpha, T) \quad J(\alpha, T)$ for all $(\alpha, T) \quad \Omega$ where $\Omega$ is the set defined in Equation (1). Furthermore, if $T$ is the minimizing final time, then $T \quad J\left(\alpha^{1}, 1\right) / C_{3}$, where $\alpha^{1}$ is an optimal control given final time $T=1$.

Proof. Given the optimal control formulation in Equations (2)-(3), suppose $(\alpha, T) \quad \Omega$ where $\Omega$ is the set defined in Equation (1). Then $\alpha$ is a vector of length $T \quad 1$ where each element of $\alpha$ is bounded between 0 and $\alpha_{\max }$. Note, the state $q_{t}$ is also bounded since $q \quad(0,1)$, $\hat{q} \quad[0,1)$, and $q_{t+1}$ is a convex combination of $q_{t}$ and $\hat{q}$, and thus $q_{t} \quad(0,1)$. Thus, for each time $T$ there exists an $\alpha^{T}$ such that $J\left(\alpha^{T}, T\right) \quad J(\alpha, T)$ for all $\alpha$ of length $T \quad 1$.

Now, suppose we create a sequence of objective functional values $J_{k}{ }_{k=1}$ where $\mathcal{J}_{k}=$ $J\left(\alpha^{k}, k\right)$, and $\alpha^{k}$ is an optimal control given final time $k$. Note that each term in the objective functional shown in Equation (2) is positive, thus $\mathcal{J}_{k}>0$ for all $k$. Additionally, for any particular $k$

$$
\mathcal{\partial}_{k}=J\left(\alpha^{k}, k\right) \quad C_{3} k \quad C_{3}
$$

for all $k$. Thus, the values of the objective functional $J(\alpha, T)$ are bounded below by $C_{3}$ for all $(\alpha, T)$, and therefore the infimum of $J(\alpha, T)$ over all pairs $(\alpha, T)$ exists. Furthermore, since $\inf _{\alpha, T} J(\alpha, T)$ exists, there exists a subsequence $\mathcal{J}_{k_{n}}=J\left(\alpha^{k_{n}}, k_{n}\right)$ such that

$$
\lim _{k_{n}} \mathcal{J}_{k_{n}}=\inf _{\alpha, T} J(\alpha, T) .
$$

Since $\mathcal{J}_{k_{n} \quad n=1}$ is a convergent subsequence it is a bounded subsequence. Thus, there exists some $M$ such that $C_{3} \quad J_{k_{n}} \quad M$. Note, by Equation (2),

$$
\mathcal{J}_{k_{n}}=C_{0}\left(\begin{array}{ll}
q_{k_{n}} & \hat{q}
\end{array}\right)^{2}+{ }_{t=0}^{k_{n} 1} C_{1} \alpha_{t}^{k_{n}}{ }^{2}+C_{2} \alpha_{t}^{k_{n}}+C_{3} k_{n}
$$

and since each term in the sum is positive, $C_{3} k_{n} \quad M$ or $k_{n} \quad M / C_{3}$. Therefore, the subsequence has a finite number of distinct terms. As a result, there exists a value $T$ $M / C_{3}$ such that $\mathcal{J}_{T^{*}}$ is the minimum of $\mathcal{J}_{k_{n}}$. Since the infimum is contained in the subsequence $\mathcal{J}_{k_{n}}$ it is contained in the sequence $\mathcal{J}_{k}$, and thus there exists a pair $(\alpha, T)$ such that $\alpha$ is an optimal control given final time $T$ and $J(\alpha, T) \quad J(\alpha, T)$ for all $(\alpha, T) \quad \Omega$.

Furthermore, when $k=T$, by Inequality (4), $C_{3} T \quad J_{T^{*}}$. Since $\mathfrak{J}_{T^{*}} \quad J_{k}$ for all $k$, $C_{3} T \quad \mathcal{J}_{T^{*}} \quad \mathcal{J}_{k}$ for all $k$, and thus

$$
T \quad \frac{\mathcal{J}_{k}}{C_{3}}=\frac{J\left(a^{k}, k\right)}{C_{3}},
$$

for all $k$. Since Inequality (5) holds for all $k$, it holds for $k=1$, and thus

$$
T \quad \frac{J\left(a^{1}, 1\right)}{C_{3}} .
$$


Though the uniqueness of optimal solutions was not shown, we point out that it can easily be shown by the structure of the objective functional that any optimal solution for which the values $\alpha_{t}$ are not identical for all $t=1, \ldots, T$, cannot be unique. For example, if an optimal solution with $T=3$ is $\alpha^{T^{*}}=[a, b, c]$, then all permutations of $[a, b, c]$ produce the same value for the objective functional, and are thus also optimal.

\subsection{Necessary Conditions}

Since the final time $T$ is a variable we derive the necessary conditions in two steps. First, supposing a given final time $T$, we determine the necessary conditions on the control $\alpha$ using a modification of Pontryagin's Minimum Principle $[8,11,17,22,25]$ and using the necessary conditions derive a characterization of the optimal control. At this point, we will have a characterization of the optimal control for any given final time $T$. Thus, we can create a sequence of optimal and controls, and thus a sequence of objective functional values for each $T=1,2, \ldots$. Assuming there exists a $T$ which minimizes the objective function, we can determine an upper bound for $T$. See [7, Chapter 8] for further details about finding an upper bound on $T$.

For a given final time $T$ we use an adaptation of Pontryagin's Minimum Principle for discrete-time difference equations $[8,11,17,22,25]$.

Theorem 2.2. Given a final time T, suppose $\alpha=\alpha_{0}, \alpha_{1}, \ldots, \alpha_{T}{ }_{1}$ is an optimal control vector containing the optimal control value $\alpha_{t}$ at each time step $t=0,1, \ldots, T \quad 1$ for the optimal control formulation in Equations (2)-(3). Let $q=\left[q_{0}, q_{1}, \ldots, q_{T}\right]$ be the corresponding state solution. Then there exists an adjoint variable such that $\lambda_{t}=\left(\begin{array}{lll}1 & \alpha_{t}\end{array}\right) \lambda_{t+1}$ and $\lambda_{T}=2 C_{0}\left(\begin{array}{ll}q_{T} & \hat{q}\end{array}\right)$. Furthermore, the optimal control is represented by

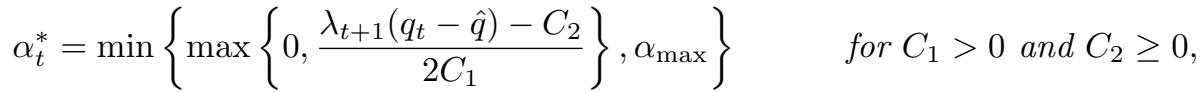

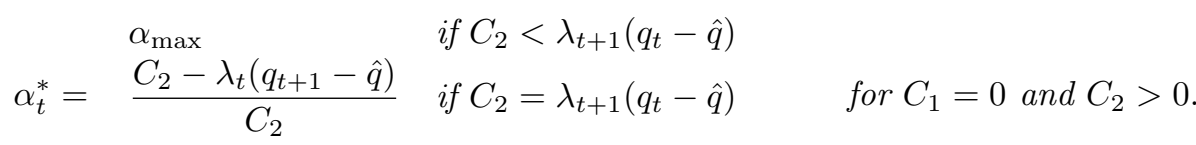

$$
\begin{aligned}
& 0 \quad \text { if } C_{2}>\lambda_{t+1}\left(q_{t} \quad \hat{q}\right)
\end{aligned}
$$

Proof. Suppose $\alpha=\alpha_{0}, \alpha_{1}, \ldots, \alpha_{T}{ }_{1}$ is a vector of optimal controls with corresponding state $q=\left[q_{0}, q_{1}, \ldots, q_{T}\right]$. Note that the objective functional in Equation (2) can be rewritten in the form

$$
J(\alpha, T)=C_{0}\left(\begin{array}{ll}
q_{T} & \hat{q}
\end{array}\right)^{2}+{ }_{t=0}^{T 1} C_{1} \alpha_{t}^{2}+C_{2} \alpha_{t}+{ }_{t=0}^{T} C_{3},
$$

and thus using Pontryagin's Minimum Principle with discrete time difference equations $[8$, $11,17,22,25]$, the Hamiltonian is

$$
H_{t}=C_{1} \alpha_{t}^{2}+C_{2} \alpha_{t}+C_{3}+\lambda_{t+1}\left[\left(1 \quad \alpha_{t}\right) q_{t}+\alpha_{t} \hat{q}\right]
$$

and the adjoint equation is

$$
\lambda_{t}=\frac{\partial H_{t}}{\partial q_{t}}=\lambda_{t+1}\left(1 \quad \alpha_{t}\right)
$$

with transversality condition giving a condition on the adjoint equation at the final time

$$
\lambda_{T}=2 C_{0}\left(q_{T} \quad \hat{q}\right) .
$$


In order to utilize Pontryagin's Minimum Principle, the Hamiltonian $H_{t}$ must satisfy the concavity condition

$$
\frac{\partial^{2} H_{t}}{\partial \alpha_{t}^{2}} \quad 0
$$

for all time steps $t$ [7]. Hence

$$
\begin{aligned}
\frac{\partial H_{t}}{\partial \alpha_{t}} & =2 C_{1} \alpha_{t}+C_{2} \quad \lambda_{t+1}\left(\begin{array}{ll}
q_{t} & \hat{q}
\end{array}\right), \\
\frac{\partial^{2} H_{t}}{\partial \alpha_{t}^{2}} & =2 C_{1} \quad 0
\end{aligned}
$$

since $C_{1} \quad 0$. Since the concavity condition is satisfied, Pontryagin's Minimum Principle tells us that the necessary conditions on $\alpha_{t}$ are

$$
\begin{array}{lll}
\alpha_{t}=\alpha_{\max } & \text { if } \frac{\partial H_{t}}{\partial \alpha_{t}}<0 \\
0 & \alpha_{t} \quad \alpha_{\max } & \text { if } \frac{\partial H_{t}}{\partial \alpha_{t}}=0 \\
\alpha_{t}=0 & & \text { if } \frac{\partial H_{t}}{\partial \alpha_{t}}>0 .
\end{array}
$$

In order to use these necessary conditions to derive a characterization of the optimal control $\alpha$, we must consider two cases: (1) $C_{1}>0$ and $C_{2} \quad 0$, and (2) $C_{1}=0$ and $C_{2}>0$.

Case 1: Let $C_{1}>0$ and $C_{2} \quad 0$. When $\frac{\partial H_{t}}{\partial \alpha_{t}}>0$, then $\alpha_{t}=0$. Thus,

$$
C_{2} \quad \lambda_{t+1}\left(q_{t} \quad \hat{q}\right)>0 \quad \frac{\lambda_{t+1}\left(q_{t} \quad \hat{q}\right) \quad C_{2}}{2 C_{1}}<0 .
$$

Note, we can divide by $2 C_{1}$ without changing the sign of the inequality because $C_{1}>0$. Lastly, when $\frac{\partial H_{t}}{\partial \alpha_{t}}<0$, then $\alpha_{t}=\alpha_{\max }$. Thus,

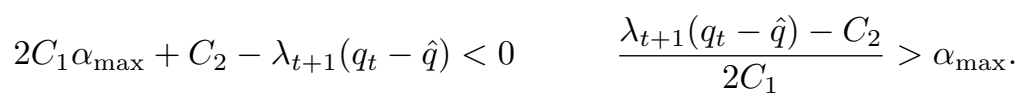

When $\frac{\partial H_{t}}{\partial \alpha_{t}}=0$, then

$$
2 C_{1} \alpha_{t}=\lambda_{t+1}\left(q_{t} \quad \hat{q}\right) \quad C_{2} \quad \alpha_{t}=\frac{\lambda_{t+1}\left(q_{t} \quad \hat{q}\right) \quad C_{2}}{2 C_{1}} \text { provided } 0<\alpha_{t}<\alpha_{\max } .
$$

Combining conditions (9)-(11), we obtain a characterization of the optimal control when $C_{1}>0$ and $C_{2} \quad 0$,

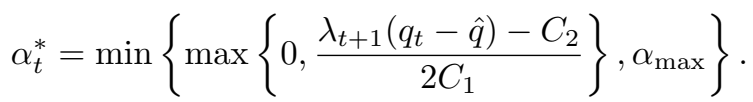

Case 2: Let $C_{1}=0$ and $C_{2}>0$, then Equation (8) becomes

$$
\frac{\partial H_{t}}{\partial \alpha_{t}}=C_{2} \quad \lambda_{t+1}\left(q_{t} \quad \hat{q}\right) .
$$

When $\frac{\partial H_{t}}{\partial \alpha_{t}}>0$, then $\alpha_{t}=0$ and $C_{2} \quad \lambda_{t+1}\left(q_{t} \quad \hat{q}\right)>0$. When $\frac{\partial H_{t}}{\partial \alpha_{t}}<0$, then $\alpha_{t}=\alpha_{\max }$ and

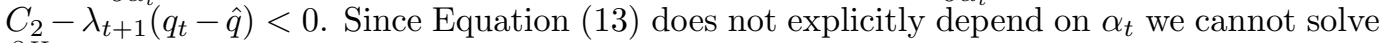
$\frac{\partial H_{t}}{\partial \alpha_{t}}=0$ for $\alpha_{t}$ as we did in Case 1. Instead, we use the state equation to rewrite the $\lambda_{t+1}$ term in Equation (13). Note, since $0<1 \quad \alpha_{t}<1$ for all $t$, we can rewrite Equation (7) as

$$
\lambda_{t+1}=\frac{\lambda_{t}}{1 \quad \alpha_{t}}
$$


Substituting the right-hand side of Equation (14) into (13) we obtain

$$
\frac{\partial H_{t}}{\partial \alpha_{t}}=C_{2} \quad \frac{\lambda_{t}}{1 \quad \alpha_{t}}\left(\begin{array}{ll}
q_{t} & \hat{q}
\end{array}\right)
$$

Setting $\frac{\partial H_{t}}{\partial \alpha_{t}}=0$ and solving for $\alpha_{t}$ we obtain

$$
\alpha_{t}=\frac{C_{2} \quad \lambda_{t}\left(q_{t} \quad \hat{q}\right)}{C_{2}} .
$$

We can verify that the $a_{t}$ in Equation (15) using the properties of the state $q_{t}$, the control $a_{t}$ and the adjoint $\lambda_{t}$. Since $0 \quad \alpha_{t} \quad \alpha_{\max }<1,0 \quad \hat{q}<1$, and $q_{0}>\hat{q}$, the sequence $q_{t} t_{t=0}$ is a decreasing sequence and $\begin{array}{llll}0 & q_{t} & \hat{q} & 1\end{array}$. By Equation (7) and the bounds on $\alpha_{t}$,

$$
\begin{aligned}
& \begin{array}{ll}
\lambda_{t} & \lambda_{t+1} \\
\hline
\end{array} \\
& \lambda_{t}\left(\begin{array}{lll}
q_{t} & \hat{q}
\end{array}\right) \quad \lambda_{t+1}\left(\begin{array}{ll}
q_{t} & \hat{q}
\end{array}\right) \\
& C_{2} \quad \lambda_{t}\left(q_{t} \quad \hat{q}\right) \quad C_{2} \quad \lambda_{t+1}\left(\begin{array}{ll}
q_{t} & \hat{q}) .
\end{array}\right.
\end{aligned}
$$

However, the right-hand side of the last inequality is zero when $\frac{\partial H_{t}}{\partial \alpha_{t}}=0$ then $C_{2}=\lambda_{t+1}\left(q_{t}\right.$ $\hat{q})$ and the last inequality becomes $C_{2} \quad \lambda_{t}\left(\begin{array}{lll}q_{t} & \hat{q}\end{array}\right) \quad \begin{array}{ll}0 . \\ \text {. }\end{array}$

Additionally, by Equation (7) and the bounds on $\alpha_{t}$,

$$
\begin{aligned}
& \left.\begin{array}{lll}
\lambda_{t} & \lambda_{t+1}(1 & \alpha_{\max }
\end{array}\right) \\
& \lambda_{t}\left(\begin{array}{ll}
q_{t} & \hat{q}
\end{array}\right) \quad \lambda_{t+1}\left(\begin{array}{lll}
q_{t} & \hat{q}
\end{array}\right)\left(\begin{array}{ll}
1 & \alpha_{\max }
\end{array}\right) \\
& C_{2} \quad \lambda_{t}\left(\begin{array}{lllll}
q_{t} & \hat{q}
\end{array}\right) \quad C_{2} \quad \lambda_{t+1}\left(\begin{array}{lll}
q_{t} & \hat{q}
\end{array}\right)\left(\begin{array}{ll}
1 & \alpha_{\max }
\end{array}\right) .
\end{aligned}
$$

However, when $\frac{\partial H_{t}}{\partial \alpha_{t}}=0$ then $C_{2}=\lambda_{t+1}\left(q_{t} \quad \hat{q}\right)$, and thus the last inequality becomes

$$
C_{2} \quad \lambda_{t}\left(q_{t} \quad \hat{q}\right) \quad C_{2} \quad C_{2}\left(1 \quad \alpha_{\max }\right)=C_{2} \alpha_{\max } .
$$

Since $C_{2}>0$, when $\frac{\partial H_{t}}{\partial \alpha_{t}}=0$ then

$$
0 \quad \frac{C_{2} \quad \lambda_{t}\left(q_{t}\right.}{\hat{q})} C_{2} \quad \alpha_{\max } .
$$

Thus,

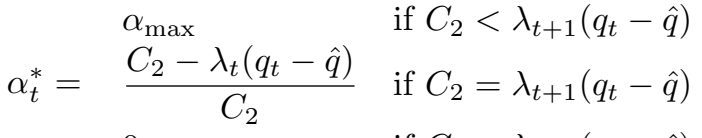

$$
\begin{aligned}
& 0 \quad \text { if } C_{2}>\lambda_{t+1}\left(\begin{array}{ll}
q_{t} & \hat{q}
\end{array}\right) \text {. }
\end{aligned}
$$

\section{Numerical Methods}

Recall the optimal solution to Equations (1)-(3) requires the minimization of the objective functional, Equation (2), over both the control $\alpha$, and the final time $T$. Each numerical optimization method we discuss in this section was a part of a meta-algorithm to find the optimal pair $\left(\alpha^{T^{*}}, T\right)$ which minimizes the objective functional $J\left(\alpha^{T}, T\right)$. Using a particular numerical optimization method, we determined the optimal control vector $\left(\alpha^{T}\right)$ and value of the objective functional $\left(J\left(\alpha^{T}, T\right)\right)$ for each final time $T=1,2, \ldots, T_{\max }$, where $T_{\max }$ is the upper bound on $T$ as defined by Inequality (6), i.e.

$$
T_{\max }=\frac{J\left(\alpha^{1}, 1\right)}{C_{3}} .
$$


This creates a sequence of values $\mathcal{J}_{T}=J\left(\alpha^{T}, T\right)$. The value of $T$ for which $\mathcal{J}_{T}$ is minimized is the minimizing final time $T$, thus yielding the optimal pair $\left(\alpha^{T^{*}}, T\right)$.

The forward-backwards sweep method is typically used to numerically determine solutions to optimal control formulation whose underlying state dynamics are in either continuous or discrete time. For a given final time, the forward-backwards sweep method starts by making an initial guess for the control vector, then solves the state equations forward in time using the state equations initial conditions. Then, using the guessed control and the newly found solution to the state equations, the adjoint equations are solved backwards in time since the adjoint equations have terminal conditions (the transversality conditions). At this point, the control vector is updating using the characterization of the optimal control and the solutions of the state and adjoint variables. This process is repeated until the successive iterates of control vectors are sufficiently close. The convergence of this iterative method for optimal control formulations whose underlying state dynamics are continuous in time is based on the work of Hackbush [9]. However, convergence for optimal control formulations whose underlying state dynamics are discrete is not guaranteed. In the case the optimal control formulation presented in Equations (1) - (3), for a given final time different initial guesses for the control vector led to different (non-optimal) solutions. Thus, we turned to other methods for numerically determining solutions.

To establish a baseline for comparing the results of any new method, we used a brute force method to determine the solutions to a small selection of parameter scenarios. For a given final time $T$, the brute force method starts by discretizing the range of the control, $\left[0, \alpha_{\max }\right]$, into $n$ subintervals creating $n+1$ possible values for each component of the control vector. We refer to $n$ as the mesh size of the discretization. Given the discretization with mesh size $n$, we next evaluate the objective functional for all possible permutations of the components of the control vector. Since the objective function depends not only on the control, but on the state variable $q$, the state equation is solved iteratively, given the control. The permutation of the control vector which minimizes the objective function is taken to be the optimal control for the given final time.

Though the brute force method is guaranteed to find the minimum value of the objective functional given a final time $T$ and discretization of mesh size $n$, the method is computationally intensive and the minimum value of the objective functional depends on the mesh size. Figure 1 shows the minimum value of the objective functional for a particular parameter set given a final time of $T=3$ over mesh sizes from $n=6$ to $n=50$. The mesh size at which the objective function is minimized (given the range of mesh sizes tested) is $n=43$.

Given that we cannot rely on the forward-backward sweep method, and the brute force method is too computationally intensive we must use alternative optimization methods that directly minimize the objective functional in Equation (2). We use the Global Optimization Toolbox from MATLAB ${ }^{\mathrm{R}}$. This toolbox offers multiple methods that search for global solutions to problems including: global search, multistart, pattern search, and genetic algorithms [19].

Optimization solvers attempt find a local optimal value for the objective functional. These algorithms locate the optimal solution in the basin of attraction of the starting point of the search. Global Optimization Toolbox solvers are intended to search in several basins of attraction, to try to find a global solution. Nevertheless, none of the solvers has an algorithm that can assure a global solution [19]. We use two different methods. The MultiStart algorithm, that uses a local solver with a broad range of starting points and the Patternsearch algorithm that searches in several basins at once, using direct search methods [19]. Nevertheless, none of the solvers has an algorithm that can assure a global solution [19].

We chose the MultiStart algorithm because it generates uniformly distributed starting points, runs all starting points, allows a choice for the local solver, and can run several starting points in parallel. These characteristics allow this algorithm to search thoroughly for a global minimum [19]. For local solver we use fmincon. When the gradient or Hessian are not provided the solver approximates the derivatives numerically. Also fmincon is one of the best options for a smooth nonlinear objective functional with bounds over the control, 


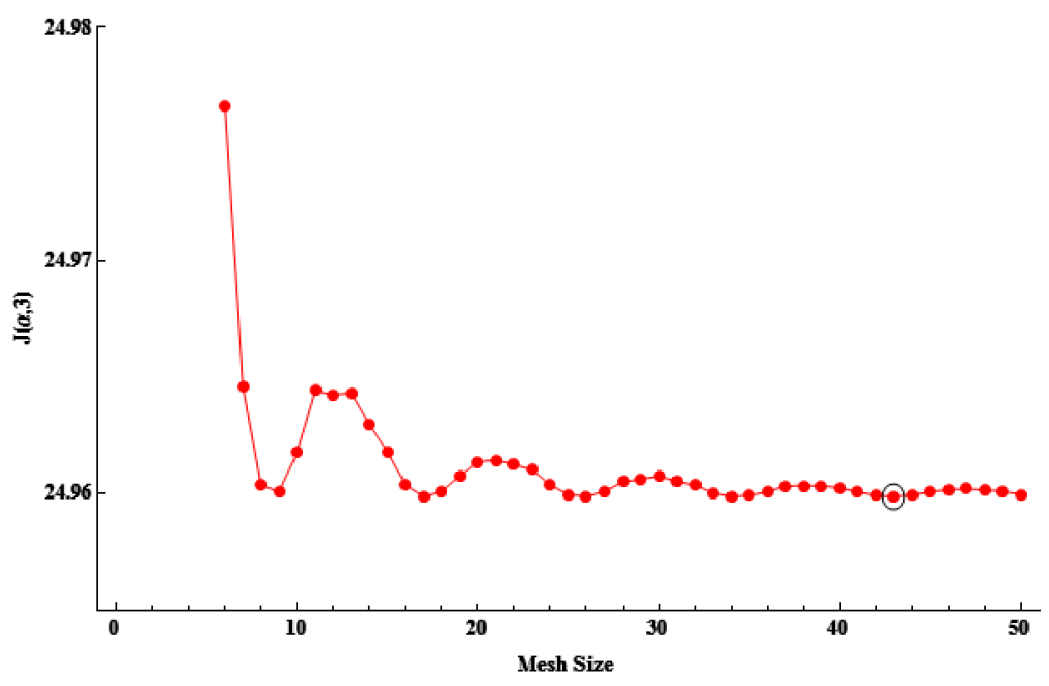

Figure 1: Value of $J\left(\alpha^{3}, 3\right)$ over different mesh sizes. Parameter values for this simulation are $q_{0}=0.997, \hat{q}=0.12547, \alpha_{\max }=0.11397, C_{0}=35.8732, C_{1}=86.6173, C_{2}=14.6181, C_{3}=0.87387$. The mesh size at which the objective function is minimized is circled.

like our objective functional. Fmincon uses a trust region approach to minimize the objective functional [20].

Alternative to MultiStart, we used Patternsearch is a direct search scheme that does not involve any information on the derivatives of the objective functional. Direct search methods search using nearby points of the current point, looking for one that has a lower value of the objective functional. This method is commonly used when the objective functional is not differentiable or not continuous [19].

The algorithm's stopping criteria limits the number of iterations in the optimization. In our case, the algorithm ends when the last step is smaller than $F$ or $X$ [20]. The value $F$ is a bound on the change in the value of the objective functional during each step. If

$$
J\left(\alpha_{i}^{T}, T-J\left(\alpha_{i+1}^{T}, T<F,\right.\right.
$$

where $\alpha_{i}^{T}$ is the control vector in iteration $i$, then the iteration ends. The bound for the size of the step is $X$, therefore the solver also stops if $[19,20]$,

$$
\alpha_{i}^{T}-\alpha_{i+1}^{T}<X
$$

For our numerical approximations we provide random starting points. For both algorithms we use $F=X=10^{-20}$. Both algorithms have a limit for the number of iterations and for the maximum number of objective functional evaluations used, and neither of those was achieved in any of our searches. In our optimal control formulation, for all parameters combinations both methods arrived to the same control solution.

\subsection{Parameter Sensitivity Analysis}

The optimal control formulation presented in Equations (1)-(3) contains the state parameters $\hat{q}$ and $q_{0}$, and the control parameters $C_{0}, C_{1}, C_{2}, C_{3}$, and $\alpha_{\max }$. Given a particular threatened population, a natural resource manager may be able to estimate $\hat{q}, q_{0}$, and $\alpha_{\max }$ with some certainty, but the exact values would not be known. Additionally, a natural resource manager might be interested in experimenting with different cost coefficients $\left(C_{0}\right.$, $\left.C_{1}, C_{2}, C_{3}\right)$ to explore the impact of changing the relative importance of each objective. Thus, there is a need for methods of exploring the sensitivity of optimal control strategies to uncertain parameter values. 
Table 1: Ranges for state and control parameters

\begin{tabular}{|c|c|c|c|}
\hline Parameter & Description & Min & $\operatorname{Max}$ \\
\hline$q_{0}$ & $\begin{array}{l}\text { initial recessive allele frequency of threatened popula- } \\
\text { tion }\end{array}$ & 0.80 & 1.00 \\
\hline$\hat{q}$ & recessive allele frequency of source population & 0.00 & 0.20 \\
\hline$\alpha_{\max }$ & $\begin{array}{l}\text { maximum proportion of threatened recessive alleles } \\
\text { which are from the source population (in each gen- } \\
\text { eration) }\end{array}$ & 0.05 & 0.20 \\
\hline$C_{0}$ & $\begin{array}{l}\text { cost coefficient; relative importance of minimizing } \\
\left(q_{T}-\hat{q}\right)^{2}\end{array}$ & 30 & 50 \\
\hline$C_{1}$ & $\begin{array}{l}\text { cost coefficient; relative importance of minimizing } \\
\text { non-linear costs associated with augmentation }\end{array}$ & 1 & 100 \\
\hline$C_{2}$ & $\begin{array}{l}\text { cost coefficient; relative importance of minimizing lin- } \\
\text { ear costs associated with augmentation }\end{array}$ & 1 & 20 \\
\hline$C_{3}$ & $\begin{array}{l}\text { cost coefficient; relative importance of minimizing the } \\
\text { final time }\end{array}$ & 0.5 & 1.5 \\
\hline
\end{tabular}

Estimates for ranges of the state and control parameters are given in Table 1. The ranges of $q_{0}$ and $\hat{q}$ were chosen to maintain the assumptions that $q_{0}$ is close to 1 , and $q_{0}>\hat{q}$. The range of $\alpha_{\max }$ was chosen to allow up to $5-20 \%$ of the threatened population in each generation to be comprised of translocated individuals. The ranges of the cost coefficients $\left(C_{0}, C_{1}, C_{2}, C_{3}\right)$ were chosen so that the relative magnitudes of the terms $C_{0}\left(q_{T} \quad \hat{q}\right)^{2}$,

${ }_{t=0}^{T}{ }^{1} C_{1} \alpha_{t}^{2}+C_{2} \alpha_{t}$, and $C_{3}(T+1)$ have the same order of magnitude. Note, if one of these three terms is orders of magnitude larger than the other two, then the relative importance of the competing objectives represented by the other two terms becomes effectively zero.

To efficiently explore the parameter space defined by the parameter ranges given in Table 1, we used Latin hypercube sampling (LHS) to sample each parameter range and generate 1000 unique parameter sets. For details about generating a Latin hypercube sample see [4]; for an example of using LHS for the uncertainty and sensitivity analysis of a system of ordinary differential equations see [3]. The optimal control solution and corresponding state solution for each of the 1000 unique parameter sets were then determined using the numerical methods described above. Recall, for the optimal control formulation given in Equations (1)(3), the optimal solution is the pair $\left(\alpha^{T^{*}}, T\right)$, where $T$ is an integer representing the minimizing final time, and $\alpha^{T^{*}}$ is an optimal control vector of length $T$. Since this approach is more computational intensive, we run our code in parallel, with four workers. For the Multistart approach we determine the number of starting points base in the length of $T$. Therefore for higher values of $T$ we use more starting points.

We examined the sensitivity of several different solution outputs to variation in the parameters. The sensitivity of the objective functional to parameter variation was determined by calculating the partial rank correlation coefficients (PRCCs) between each of the parameters (using uniform probability density functions over the ranges given in Table 1) and the value of the objective functional at the optimal solution. The magnitude of each PRCC value indicates the strength of linear correlation between the parameter value and objective functional value, while the sign of each PRCC value shows whether the correlation is positive or negative. Table 2 shows the resulting PRCC values.

To determine the sensitivity of the minimizing final time to parameter variation, we first categorize the optimal solution for each parameter set by its minimizing final time $T$. Then for each parameter, we generate box plots and density plots of each parameter value over the range of minimizing final times. These plots reveal to which parameter values the minimizing final time is most sensitive. 
Table 2: Sensitivity of the objective functional to parameter variation using Partial Rank Correlation Coefficients (PRCCs).

\begin{tabular}{cc}
\hline Parameter & $\begin{array}{c}\text { PRCC } \\
\text { Value }\end{array}$ \\
\hline$\hat{q}$ & -0.9308 \\
\hline$q_{0}$ & 0.8920 \\
\hline$C_{0}$ & 0.8881 \\
\hline$C_{2}$ & 0.7050 \\
\hline$C_{3}$ & 0.6811 \\
\hline$C_{1}$ & 0.2484 \\
\hline$\alpha_{\max }$ & -0.1968 \\
\hline
\end{tabular}

To determine the sensitivity of the optimal control vector $\alpha^{T^{*}}$ to parameter variation, we start by categorizing the optimal control vector based on qualitative traits. In all of our numerical simulations the optimal augmentation strategy was augment with the same proportion at each time step, i.e. $\alpha_{t}$ is the same for all $t=1, \ldots, T$. Thus, for simplicity in this analysis, we divide optimal control solutions into two categories: (1) $\alpha_{t}=\alpha_{\max }$ for all $t$, and (2) $\alpha_{t}<\alpha_{\max }$ for all $t$. Note, the first strategy is to apply the maximum amount of control at every time step, where as the second category is to apply a lesser amount of control at each time step. As with the minimizing final time, for each parameter we then generate box plots and density plots of each parameter value over each qualitative optimal control strategy.

\section{Numerical Results}

In numerically solving for the optimal solution to Equations (1)-(3), we find the optimal solution given each final time $T=1,2, \ldots, T_{\max }$, where $T_{\max }$ is given in Equation (16). Before we performed the uncertainty and sensitivity analysis, we examined how the final time changed the value of the objective functional for a few select parameter sets. Figure 2 show the value of $J\left(\alpha^{T}, T\right)$ for values of $T=1,2, \ldots, T_{\max }$ given parameter values $q_{0}=0.997$, $\hat{q}=0.12547$ (in Figure 2a), $\alpha_{\max }=0.11397, C_{0}=35.8732, C_{1}=86.6173, C_{2}=14.6181$, $C_{3}=0.87387$ (in Figure 2b). In Figure 2a, the value of $C_{3}$ is varied from 0.5 to 2.0, and in Figure $2 \mathrm{~b}$ the value of $\hat{q}$ is varied from 0.01 to 0.25 . In each parameter scenario, we see that the value of $T_{\max }$ is much larger than the value of $T$, the minimizing final time shown as the circled point on each curve. Additionally, we see that as the value of $C_{3}$ is increased, the value of the objective functional increases, but as the value of $\hat{q}$ is increased, the value of the objective functional decreases. Note, all parameters other than $C_{3}$ in Figure 2a and $\hat{q}$ in Figure $2 \mathrm{~b}$ remained unchanged. Figure 3 shows how the value of $T_{\max }$ decreases as the values of $C_{3}$ and $\hat{q}$ are increased for the parameter scenarios shown in Figure 2. Note, $T_{\max }$ appears to decay linearly with respective to $\hat{q}$, but nonlinearly with respect to $C_{3}$. This is likely due to the fact that $T_{\max }$ is defined as a fraction whose denominator is $C_{3}$.

To efficiently examine the sensitivity of the objective functional, minimizing final time, and optimal control strategy to variation in uncertain parameters, we numerically solved Equations (1)-(3) for the optimal solution for each of the 1000 unique parameter sets generated using the LHS method given the parameter ranges defined in Table 1. The sensitivity of the objective functional to parameter uncertainty is shown in Table 2 by the values of the PRCCs between each of the parameters and the value of the objective functional at the optimal solution. Note the correlation between $C_{3}$ and the objective functional is positive, 
and the correlation between $\hat{q}$ and the objective functional is negative, as seen in the selective set of parameter scenarios in Figure 2. Thus, as the relative importance of minimizing the final time $\left(C_{3}\right)$ is increased, the value of the objective functional is increased. However, as the frequency of the recessive allele in the source population is increased, the value of the objective functional is decreased.

We define the objective functional as having little sensitivity to parameters with PRCC values whose magnitudes is less than 0.5 , moderate sensitivity to parameters with PRCC values whose magnitude is between 0.5 and 0.8 , and high sensitivity to parameters with PRCC values whose magnitude is above 0.8. Thus, we see that the objective functional has high sensitivity to parameters $\hat{q}, q_{0}$, and $C_{0}$. This means that small increases in these parameter values will cause large changes in the value of objective functional (increases if the sign of the PRCC value is positive, and decreases if the sign of the PRCC value is negative). The objective functional has low sensitivity to $C_{1}$ and $\alpha_{\max }$, which means that small increases in these parameter values will cause only small scale changes in the value of the objective functional.

Since the minimizing final time $(T)$ can only take on discrete integer values, we cannot use PRCCs to measure the sensitivity of $T$ to parameter uncertainty. Instead, we graphically display the range of parameter values resulting in each minimizing final time (see Figure 2). Over the 1000 unique parameter sets used, the distribution of minimizing final times is heavily skewed to the left, as shown in Figure 4. In fact, minimizing final times above 7 account for only five of the 1000 simulations. Note, that roughly one-third of the simulations resulted in a minimizing final time of $T=1$, which means there is only one time step at which the control (the augmentation strategy) can be applied.

Figures 5a-5e show the sensitivity of $T$ to uncertainty in various parameters. In each graph, the value of the parameter in each of the 1000 parameter sets is plotted on the vertical axis. The horizontal axis shows the corresponding values of $T$ for each parameter set. For each $T$ a density plot shows value of the parameter for each parameter set whose minimizing final time is $T$. The overlayed grey region shows the interquartile range of parameter values with that minimizing final time, the red line shows the median value, and the blue diamond shows the mean value. The number above each density plot indicates the number of parameter sets which resulted in the corresponding $T$ value, and correlates to Figure 4. Since so few parameter sets results in $T=8,9$, 10, or 11, no interquartile range, median or mean values are shown in these cases. It should be noted that though each graph is showing the variation in a single parameter, all the parameters are varying

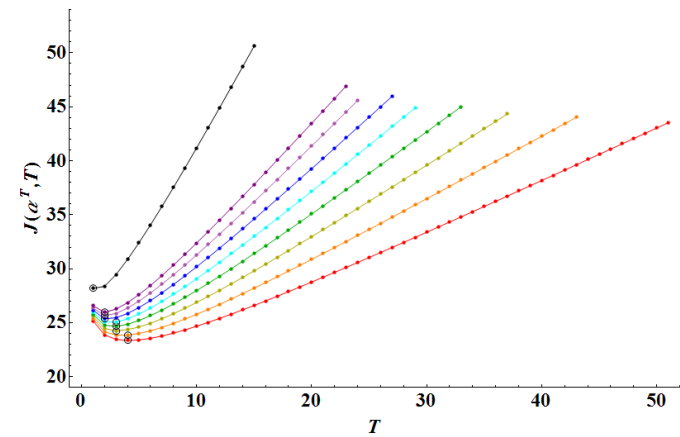

(a) $C_{3}=0.5$ (red), 0.6 (orange), 0.7 (yellow), 0.8 (green), 0.9 (cyan), 1.0 (blue), 1.1 (light purple), 1.2 (dark purple), 2.0 (black).

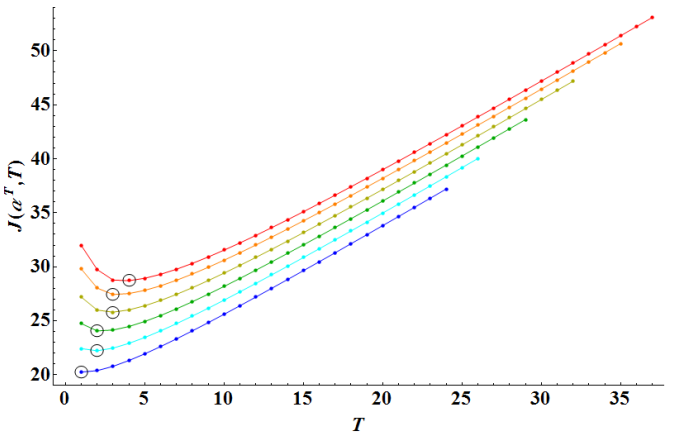

(b) $\hat{q}=0.01$ (red), 0.05 (orange), 0.10 (yellow), 0.15 (green), 0.20 (cyan), 0.25 (blue).

Figure 2: Value of $J\left(\alpha^{T}, T\right)$ for values of $T=1,2, \ldots, T_{\max }$. Parameter values for each simulation are $q_{0}=0.997, \hat{q}=0.12547$ (in (a)), $\alpha_{\max }=0.11397, C_{0}=35.8732, C_{1}=86.6173, C_{2}=14.6181$, $C_{3}=0.87387$ (in (b)). The mesh size at which the objective function is minimized for each parameter value is circled. 


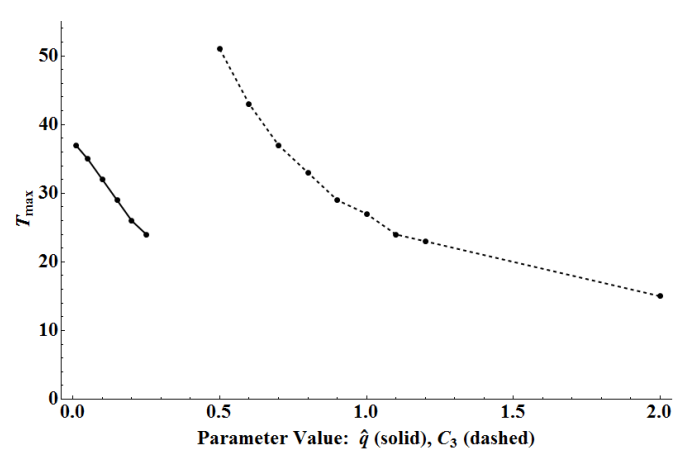

Figure 3: Value of $T_{\max }$ given varying values of $\hat{q}$ and $C_{3}$. Parameter values for each simulation are $q_{0}=0.997, \hat{q}=0.12547$ (when $C_{3}$ varies, dashed), $\alpha_{\max }=0.11397, C_{0}=35.8732, C_{1}=$ 86.6173, $C_{2}=14.6181, C_{3}=0.87387$ (when $\hat{q}$ varies, solid). The value of $T_{\max }$ is determined by Equation (16).

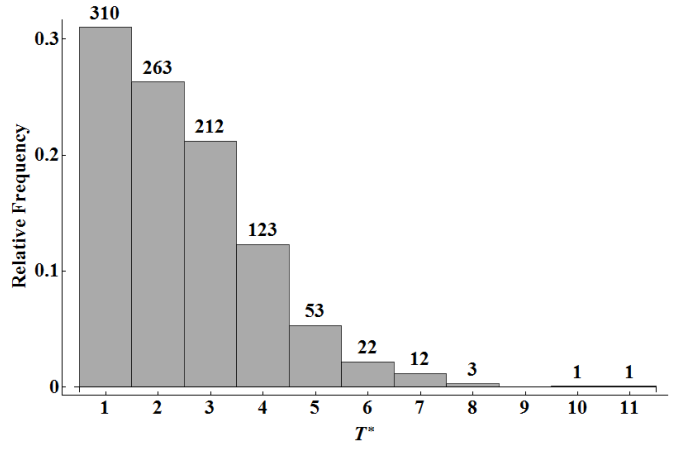

Figure 4: The relative frequency of minimizing final times over the 1000 unique parameter sets generated using LHS and uniform probability density functions over the parameter ranges given in Table 1 . The number above each bar indicates the number of simulations resulting in the corresponding minimizing final time.

simultaneously.

The visual display of the parameter values which correspond particular values of $T$ make it easier to see trends. For example, we can see that as the values of $C_{0}$ (see Figure 5a) and $q_{0}$ (see Figure 5a) increase, we would on average expect the minimizing final time to increase. From Figure 5 we can also see that the as the values of $C_{2}$ (see Figure $5 \mathrm{~b}$ ), $C_{3}$ (see Figure 5c), and $\hat{q}$ (see Figure 5e) increase, we would on average expect the minimizing final time to decrease. However, since the interquartile ranges on each of these graphs heavily overlap, the correlations are not strong. The sensitivities of $T$ to uncertainty in $C_{1}$ and $\alpha_{\max }$ are not shown because virtually no correlation existed (all interquartile ranges, median and mean values were roughly the same over all values of $T$ ).

Figure $5 \mathrm{f}$ shows the sensitivity of the optimal control strategy $\left(\alpha^{T^{*}}\right)$ to uncertainty in the parameter $\alpha_{\max }$. The structure of the graph is similar to those of Figures 5a-5e. However, instead of categorizing the optimal solution for each of the 1000 unique parameter sets by the value of $T$, we have divided the solutions into two qualitative control strategies. For each of the 1000 parameter sets, the optimal augmentation strategies was to augment with the same proportion at each time step, i.e. $\alpha_{t}$ is the same for all $t=1, \ldots, T$. Thus, the two qualitative strategies we defined where (1) $\alpha_{t}=\alpha_{\max }$ for all $t$, and (2) $\alpha_{t}<\alpha_{\max }$ for all $t$. From Figure $5 \mathrm{f}$ we see that smaller values of $\alpha_{\max }$ correspond with the optimal control strategy being to apply the maximum value of the control at every time step, i.e. maximally augment the threatened population at each time step. When the value of $\alpha_{\max }$ is larger, we expect on average the optimal strategy to be to apply some amount of control below the maximum allowable amount. Notice that in Figure $5 \mathrm{f}$ the interquartile ranges do not overlap, and thus we classify this correlation as stronger than the correlations shown Figures $5 \mathrm{a}-5 \mathrm{e}$. The sensitivities of the qualitative control strategies to uncertainty in all other parameters are not shown because virtually no correlation existed (all interquartile ranges, median and mean values were roughly the same over both qualitative control strategies).

\section{Conclusions}

The underlying model for the optimal control formulation, the Continent-Island model was chosen for its simplicity so that we might demonstrate this novel process of parameter sensitivity analysis applied to an optimal control problem. Despite the simplicity of the underlying model for the state variable, the parameter sensitivity analysis revealed trends 


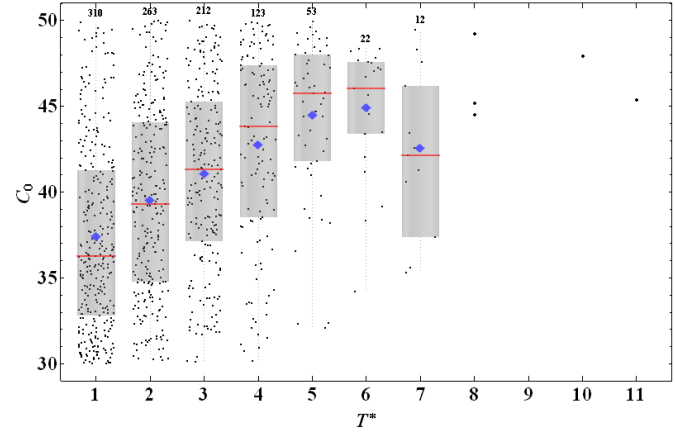

(a) $T^{*}$ sensitivity to $C_{0}$.

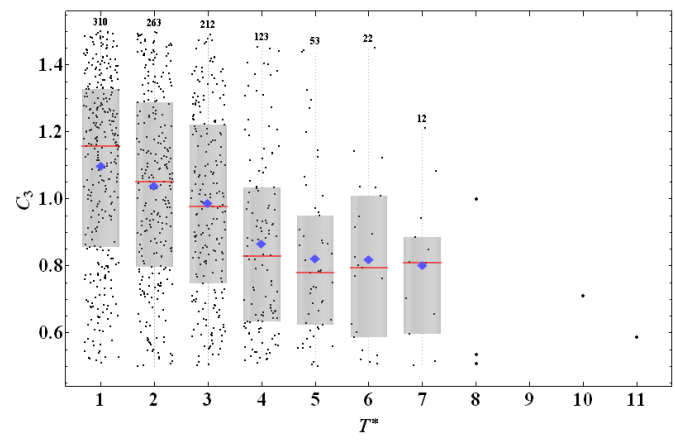

(c) $T^{*}$ sensitivity to $C_{3}$.

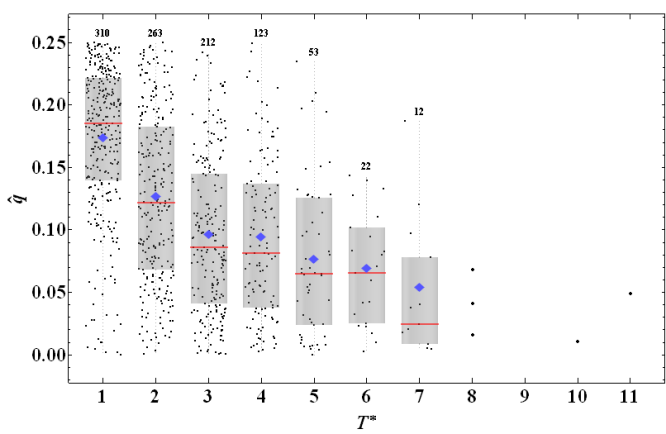

(e) $T^{*}$ sensitivity to $\hat{q}$.

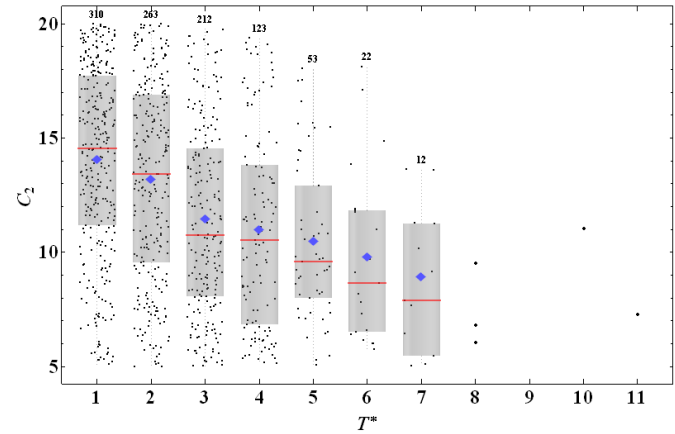

(b) $T^{*}$ sensitivity to $C_{2}$.

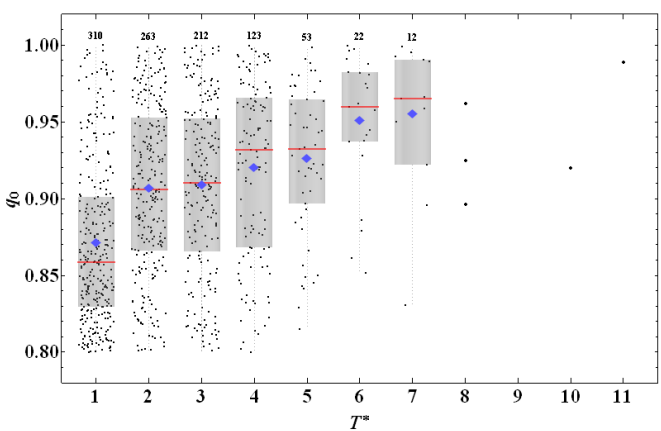

(d) $T^{*}$ sensitivity to $q_{0}$.

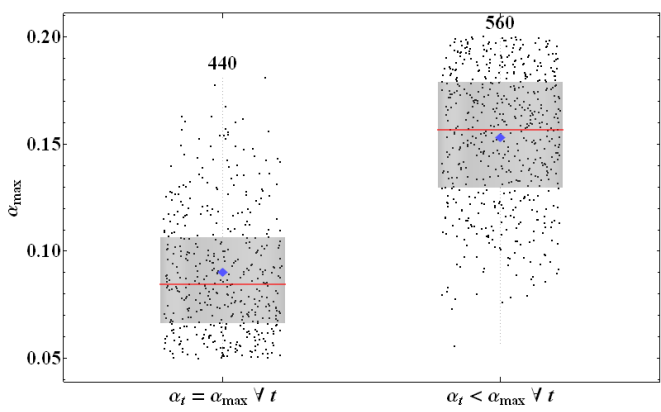

(f) Qualitative control strategy sensitivity to $\alpha_{\max }$.

Figure 5: The sensitivity of $T^{*}$ and qualitative control strategies to uncertainty in various parameters. In each graph, the value of the parameter in each of the 1000 parameter sets is plotted on the vertical axis. The density plots show the range of the parameter values for each minimizing final time (graphs (a)-(e)), and for each qualitative control strategy (graph (f)). The shaded grey regions show the interquartile ranges of the for each qualitative strategy with the red lines showing the median values, and the blue diamonds showing the mean values. The numbers above each density plot indicate the number of parameter sets which resulted in the corresponding minimizing final time or optimal qualitative control strategy. 
in the optimal augmentation strategies which would not necessarily be evident by testing a few select parameter sets. For example, $C_{3}$ is the cost coefficient representing the relative importance of minimizing the final time. As one would expect, increasing the value of $C_{3}$ lowers, on average, the expected minimizing final time (see Figure 5c). However, increasing the value of $C_{3}$ increases, on average, the expected value of the objective functional. Since the value of the objective functional at the optimal solution represents the relative total cost of implementing that optimal strategy, we see that there is a trade-off between implementing a genetic augmentation quickly and implementing an genetic augmentation at a lower cost. As another example, $\hat{q}$ is the (constant) frequency of the recessive allele in the source population. From the sensitivity analysis we see that both the value of the objective functional and the minimizing final time are negatively correlated to $\hat{q}$ (see Table 2 and Figure 5e, respectively). In fact, the value of the objective functional is most sensitive to changes in $\hat{q}$. However, the qualitative control strategy is not sensitive to the value of $\hat{q}$. Thus, if resource managers are using these types of analyses to inform their augmentation strategies, it would be important to have a good measurement of $\hat{q}$ for the gene in which they are interested if they want to compute realistic values of the minimizing final time and value of the objective functional (relative cost).

It should be noted that Miller Neilan et al. used parameter sensitivity analysis in [21] to inform the construction of the optimal control formulation for cholera intervention. However, this is different from the method of parameter sensitivity analysis we developed which evaluates the sensitivity of the optimal solution and value of the objective functional.

If this optimal control formulation is to be used to inform the augmentation strategies of a specific species, the values of the cost coefficients could be modified to reflect actual dollar amounts so that the value of the objective functional represented a true total cost. Additionally, note that the value of the control at each times step, $\alpha_{t}$ is the proportion of the threatened population at generation $t$ which are translocated from the source population. The for actual implementation, these proportions would need to be converted to numbers of individuals. One extension of this optimal control formulation would be to change the underlying state equation to a discrete difference equation system which models the population size or density (as Bodine et al. presented in [6]), but which additionally tracks a measure of genetic fitness within the population.

Typically, resource managers designing genetic augmentations would have a limited set of feasible strategies to consider. Ultimately, this optimization approach aims to provide a means of comparing genetic augmentation strategies given uncertain parameters, and our method of parameter sensitivity analysis enables resource managers to efficiently test a wide range of cost coefficients and determine the span of possible "optimal" augmentation strategies given that range.

\section{Acknowledgements}

This work was assisted by attendance of ENB as a Short-term Visitor, and by MVM as a Graduate Fellow at the National Institute for Mathematical and Biological Synthesis (NIMBioS), an Institute sponsored by the National Science Foundation, the U.S. Department of Homeland Security, and the U.S. Department of Agriculture through NSF Award \#EF0832858, with additional support from the University of Tennessee, Knoxville (UTK). Both authors would like to thank Suzanne Lenhart at UTK and NIMBioS for her valuable comments on this project, and for suggesting the collaboration of ENB and MVM that led to the successful completion of this research. Lastly, this work was additionally supported in part by funding from a Faculty Development Grant award to ENB through Rhodes College. 


\section{References}

[1] K. Alvarez. Twilight of the Panther: Biology, Bureaucracy and Failure in an Endangered Species Program. Myakka River, Sarasota, Florida, 1993.

[2] C. Belden and K. Warren. Florida Panther and the Genetic Restoration Program. Technical report, U.S. Fish \& Wildlife Service, 2008.

[3] S. M. Blower, E. N. Bodine, and K. Grovit-Ferbas. Predicting the potential public health impact of disease-modifying HIV vaccines in South Africa: The problem of subtypes. Current Drug Targest - Infectious Disorders, 5:179-192, 2005.

[4] S. M. Blower and H. Dowlatabadi. Sensitivity and uncertainty analysis of complex models of disease transmission: An HIV model, as an example. International Statistical Review, 62(2):229-243, 1994.

[5] E. N. Bodine, L. J. Gross, and S. Lenhart. Optimal control applied to a model for species augmentation. Mathematical Biosciences and Engineering, 4(5):669-680, 2008.

[6] E. N. Bodine, L. J. Gross, and S. Lenhart. Order of events matter: Comparing discrete models for optimal control of species augmentation. Journal of Biological Dynamics, 6(Supplement 2):31-49, 2012.

[7] M. D. Canon, C. D. Cullum, and E. Polak. Theory of Optimal Control and Mathematical Programming. McGraw-Hill, New York, 1970.

[8] C.W. Clark. Mathematical Bioeconomics: The Optimal Management of Renewable Resources. Wiley-Interscience, $2^{\text {nd }}$ edition, 1990.

[9] W. Hackbusch. A numerical method for solving parabolic equations with opposite orientations. Computing, 20(3):229-40, 1978.

[10] A. M. Haines, M.E. Tewes, L.L. Laack, J.S. Horne, and J.H. Young. A habitatbased population viability analysis for ocelots (leopardus pardalis) in the united states. Biological Conservation, 132(4):424-436, October 2006.

[11] H. Halkin. A maximum principle of the Pontryagin type for systems described by nonlinear difference equations. SIAM Journal on Control, 4(1):90-111, 1966.

[12] J. W. Hearne and J. Swart. Optimal translocation strategies for saving the black rhino. Ecological Modelling, 59(3-4):279-292, December 1991.

[13] P. W. Hedrick. Gene flow and genetic restoration: The Florida panther as a case study. Conservation Biology, 9(5):996-1007, 1995.

[14] P. W. Hedrick. Genetics of Populations. Jones and Bartlett Publishers, Boston, $4^{\text {th }}$ edition, 2011.

[15] N. Kingston, S. Waldren, and N. Smyth. Conservation genetics and ecology of angiopteris chauliodonta copel. (marattiaceae), a critically endangered fern from pitcairn island, south central pacific ocean. Biological Conservation, 117(3):309-319, May 2004.

[16] D. Land, M. Cunningham, M. Lotz, and D. Shindle. Florida panther annual report 2004-05. Technical report, Florida Fish and Wildlife Conservation Commission, Tallahassee, Florida, November 2005.

[17] S. Lenhart and J. T. Workman. Optimal Control of Biological Models. Chapman and Hall/CRC Publishers, Boca Raton, 2007. 
[18] D. Maes, W. Vanreusel, W. Talloen, and H. Van Dyck. Functional conservation units for the endangered alcon blue butterfly maculinea alcon in belgium (lepidoptera: Lycaenidae). Biological Conservation, 120(2):229-241, November 2004.

[19] The MathWorks, Inc. Global Optimization Toolbox User's Guide, revised for version 6.3 (release 2013a) edition, March 2013.

[20] The MathWorks, Inc. Optimization Toolbox User's Guide, revised for version 6.3 (release 2013a) edition, March 2013.

[21] R. L. Miller Neilan, E. Schaefer, H. Gaff, K. R. Fister, and S. Lenhart. Modeling optimal intervention strategies for Cholera. Bulletin of Mathematical Biology, 72:2004-2018, 2010 .

[22] J. A. Ortega and R. J. Leake. Discrete maximum principle with state constrained control. SIAM Journal on Control and Optimization, 15(6):984-990, 1977.

[23] S. L. Pimm, L. Dollar, and O.L. Bass Jr.. The genetic rescue of the florida panther. Animal Conservation, 9:115-122, 2006.

[24] K. A. Romain-Bondi, R. B. Wielgus, L. Waits, W.F. Kasworm, M. Austin, and W. Wakkinen. Density and population size estimates for north cascade grizzly bears using dna hair-sampling techniques. Biological Conservation, 117(4):417-428, June 2004 .

[25] S. P. Sethi and G.L. Thompson. Optimal control theory: applications to management science and economics. Springer, $2^{\text {nd }}$ edition, 2005.

[26] S. Wright. Evolution in Mendelian Populations. Genetics, 16:97-159, 1931. 